\title{
L'ENSEIGNEMENT DU NUCLÉAIRE EN FRANCE
}

C e n'est pas aux lecteurs de la RGN que l'on apprendra que l'industrie nucléaire française est, depuis quelques années, en plein essor. $\mathrm{Si}$, au départ, elle a bénéficié d'un potentiel scientifique d'une qualité exceptionnelle, elle a maintenant besoin d'un nombre accru d'ingénieurs et de techniciens hautement qualifiés. Où ces derniers peuvent-ils recevoir la formation nécessaire? Notre revue a décidé de consacrer ce numéro à une présentation de l'enseignement du nucléaire en France.

A notre surprise, il est vite apparu que très peu de formations existaient dans ce domaine, notamment à l'université. S'il est fait parfois appel à des conférenciers pour traiter d'un sujet en particulier, il n'existe que très peu de cours « nucléaires » sanctionnés par un examen. Un exemple parmi tant d'autres : l'enseignement de la radiochimie, qui n'était plus dispensé qu'à Paris VI où il s'inscrivait en succession de l'ancienne chaire des Joliot-Curie, a disparu de cette université qui s'appelle pourtant «Université Pierre et Marie Curie »! Le seul grand établissement qui délivre des diplômes dans ce domaine reste le Conservatoire National des Arts et Métiers (CNAM), mais chacun connaît bien la spécificité de cet enseignement réservé à la promotion supérieure du travail. L'autre grand organisme de formation est l'Institut National des Sciences et Techniques Nucléaires (INSTN) avec ses sessions d'études, ses stages de formation continue et ses cours de génie atomique.
Q $n$ peut s'interroger sur les motifs de cette désaffection, qui est peut-être passagère, et même s'en étonner. Il n'en reste pas moins qu'elle est préjudiciable à l'industrie nucléaire qui éprouve quelque difficulté à trouver, sur le marché de l'emploi, un nombre suffisant d'ingénieurs et de techniciens rompus à ces techniques nouvelles.

C e numéro de la RGN, s'il n'a pas l'ambition de dresser une liste exhaustive des formations existantes, aura toutefois eu le mérite de mettre l'accent sur cet aspect du problème... et de souligner par ailleurs la qualité des trop rares enseignements dispensés dans ce domaine.

Jacques FOOS 